Supplementary Information

\title{
Control and characterization of the compactness of single-chain nanoparticles
}

Suiyang Liao*, ${ }^{*}$ Lixia Wei, ${ }^{a}$ Luciano A. Abriata, ${ }^{c}$ Francesco Stellacci ${ }^{* a, b}$

*E-mail: suiyang.liao@epfl.ch

*E-mail: francesco.stellacci@epfl.ch

a. Institute of Materials, École Polytechnique Fédérale de Lausanne, Station 12, 1015 Lausanne, Switzerland

b. Interfaculty Bioengineering Institute, École Polytechnique Fédérale de Lausanne, Station 12, 1015 Lausanne, Switzerland

c. Protein Production and Structure Core Facility, School of Life Sciences, École Polytechnique Fédérale de Lausanne, $\mathrm{CH}-1015$, Lausanne, Switzerland 


\section{Table of Contents}

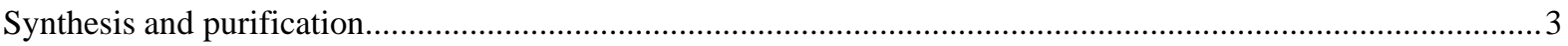

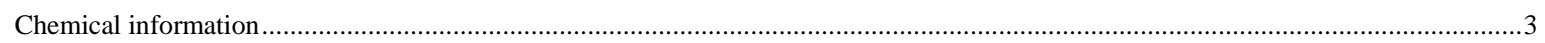

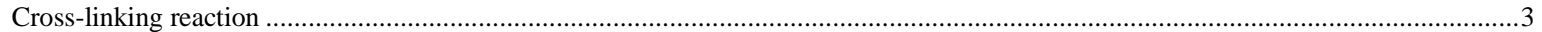

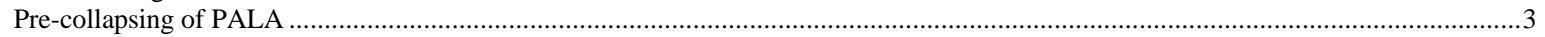

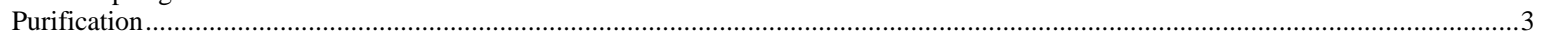

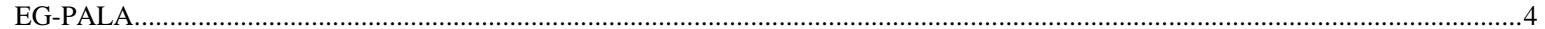

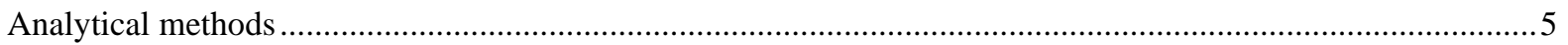

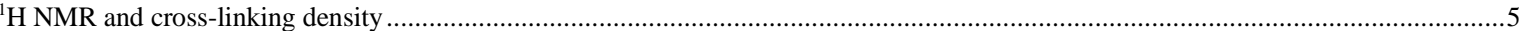

DOSY NMR

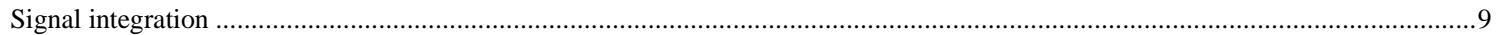

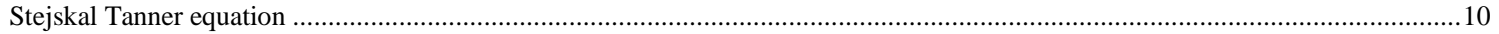

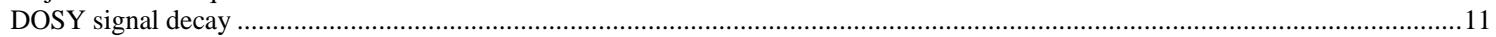

Viscometry ...

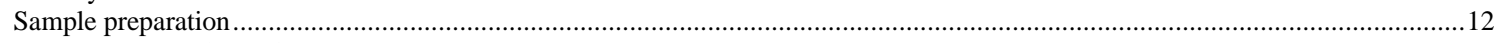

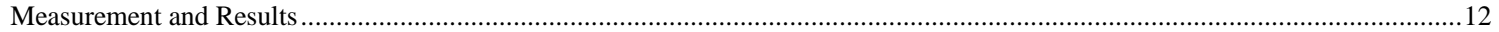

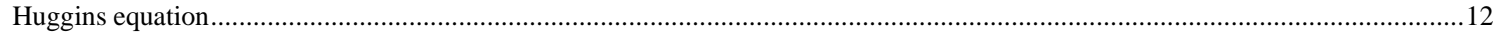

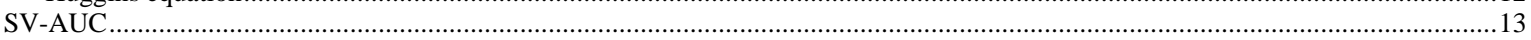

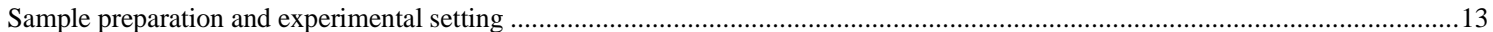

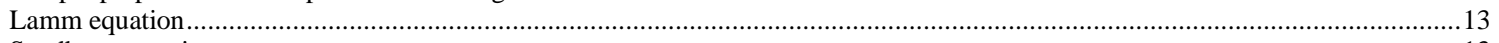

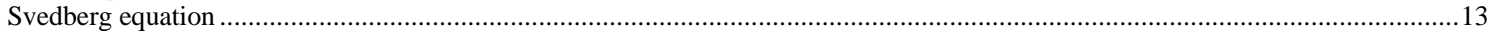

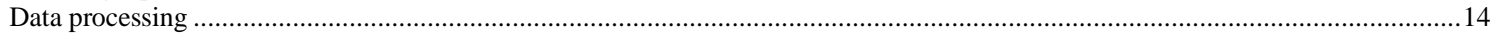

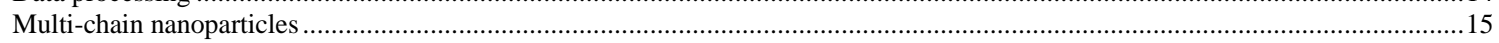

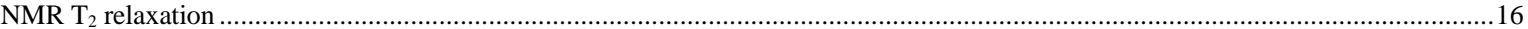

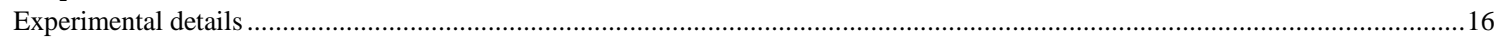

GPC

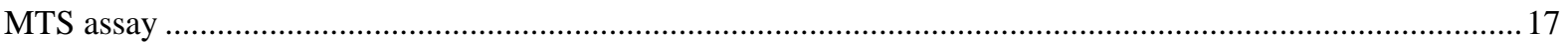

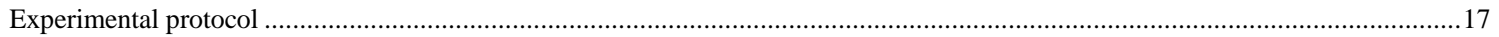

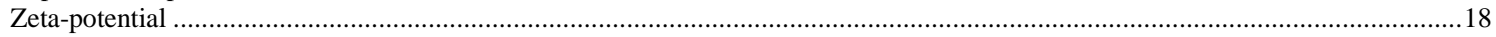

MTS plot with PALA and EG2-PALA

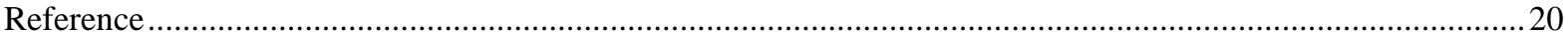

\section{The List of Figures, Tables and Equations in this document:}

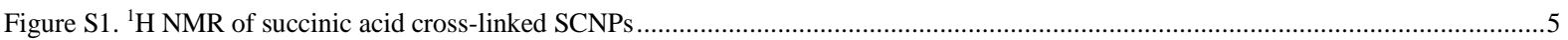

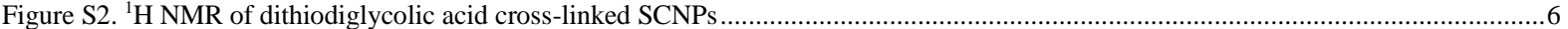

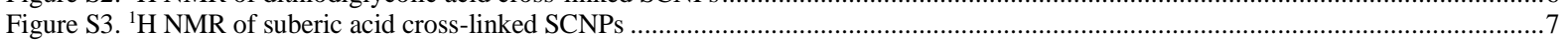

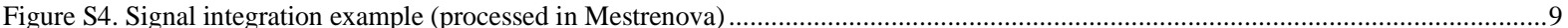

Figure S5. Scatter plots showing DOSY signal decay. (cross-linkers are succnic acids, dithiodiglycolic acids, and suberic acids, respectively

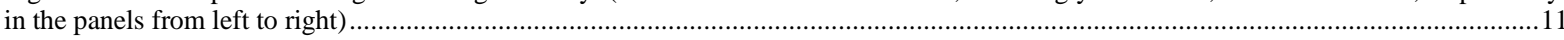

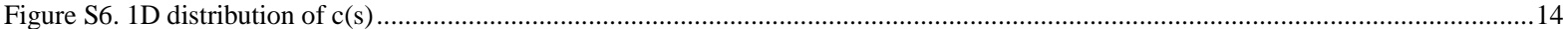

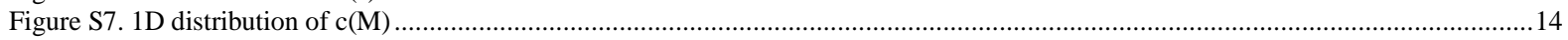

Figure S8. Distribution of sedimentation coefficient on SCNPs and multi-chain NPs (cross-linked above the critical concentration) ...........15

Figure S9. Scatter plot with all SCNPs and reference materials' zeta potential results (plotted as mean \pm SD) .......................................18

Figure S10. Chemical structure and proton NMR of EG-PALA

Figure S11. HeLa cell viability via MTS assay upon 24-hour incubation with all samples. (black circle: PALA; grey circle: EG-PALA).

Each data point represents three independent experiments and reported as mean \pm SD. ......................................................................19

Table S1. Relative viscosity $\eta / \eta_{0}$ 


\section{Synthesis and purification}

\section{Chemical information}

PALA (Polyallylamine hydrochloride, CAS 71550-12-4), succinic acid (CAS 110-15-6), dithiodiglycolic acid (CAS 505-73-7), suberic acid (CAS 505-48-6), EDC (CAS 22572-40-3), and NHS (6066-82-6) were purchased from Sigma-Aldrich and used without further purification.

\section{Cross-linking reaction}

$150 \mathrm{mg}$ parent polymer PALA was dispersed into $150 \mathrm{ml}$ chosen reaction conditions as discussed in the paper to form dilute precursor solution. To crosslink $50 \%$ of the primary amines, around $96 \mathrm{mg}$ succinic acids ( 2 molar excess to $50 \%$ of the amines) were dissolved into $10 \mathrm{ml}$ corresponding buffer, followed with the addition of EDC and NHS (both at 1.5 molar excess to carboxylic groups) for activation and stabilization. The activated crosslinker solution was then transferred into an additional funnel. While stirring the precursor solution in a $250 \mathrm{ml}$ round bottom flask, the crosslinker solution was added dropwise at $\sim 1$ drop/s. The reaction was kept at room temperature overnight.

\section{Pre-collapsing of PALA}

To show the solvent-induced compaction of PALA, DLS was performed in the corresponding solvent conditions. Figure S1 shows size distribution by volume (with viscosity correction).

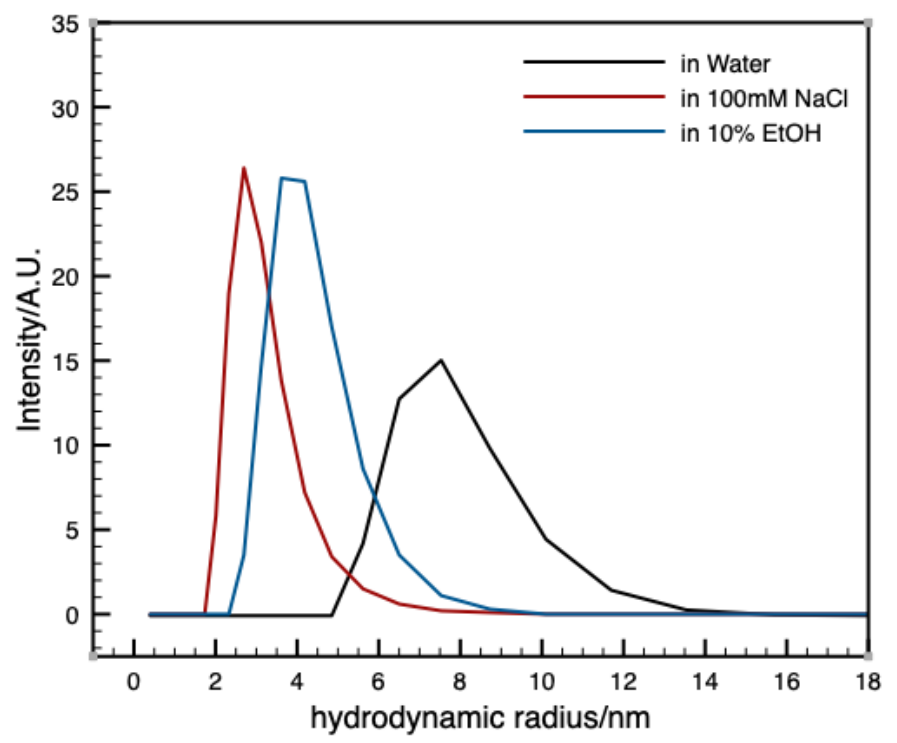

Figure S1. DLS size distribution (by volume) of PALA in different solvent conditions.

\section{Purification}

Ultrafiltration was performed on the reaction mixture with Amicon® Ultra-15 filter (Mw cutoff: 3,000 Da) to concentrate the final product, which was later washed with ultrapure water for multiple rounds to desalt. The concentrated sample underwent extensive dialysis in ultrapure water before the final concentrating step. Finally, the sample was freeze dried into dry powder. 


\section{EG-PALA}

The structure-cytotoxicity relationship was also evidence by conjugating the PALA chain with 2-[2-(2Methoxyethoxy)ethoxy]acetic acid via amidation, to yield a similar ratio of free primary amine groups. The proton NMR is shown in Figure S2. The final product is referred as EG-PALA. It shows similar zeta-potential profile to the SCNPs in the work. (Figure S2)

A)
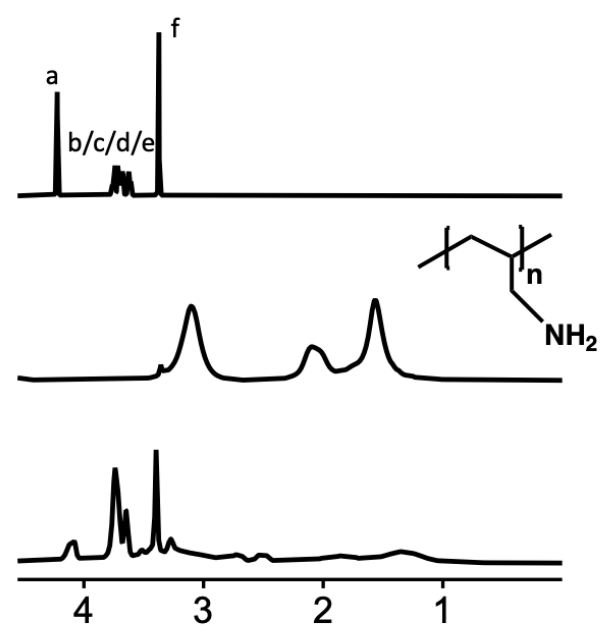

B)<smiles>CCC(C)(C)CC(C)(C)C(C)CNC(=O)CCl</smiles>
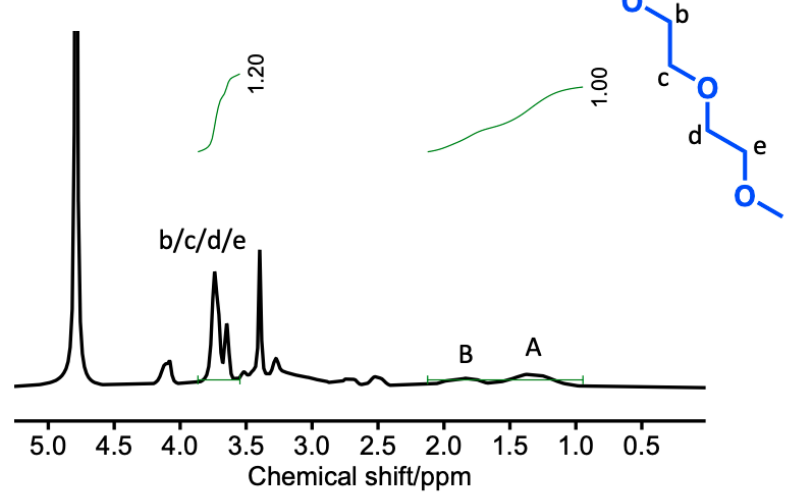

Figure S2. A) proton NMR of (from top to bottom) 2-[2-(2-Methoxyethoxy)ethoxy]acetic acid; PALA; EG-PALA; B) Ligand conjugation ratio is quantified to be $40 \%$, comparable to the cross-linking ratio of the SCNPs in this work 


\section{Analytical methods}

\section{${ }^{1}$ H NMR and cross-linking density}

${ }^{1} \mathrm{H}$ NMR spectra were obtained using excitation sculpting centered on the water resonance in spectral widths of around $14 \mathrm{ppm}$ wit 16k points. Resonance assignments were straightforward from chemical shifts of the ${ }^{1} \mathrm{H}$ resonances. The cross-linking density was quantified as the ratio of the amide (quantified by the signal from the cross-linker protons) to the initial primary amines (quantified by the signal from the backbone protons).

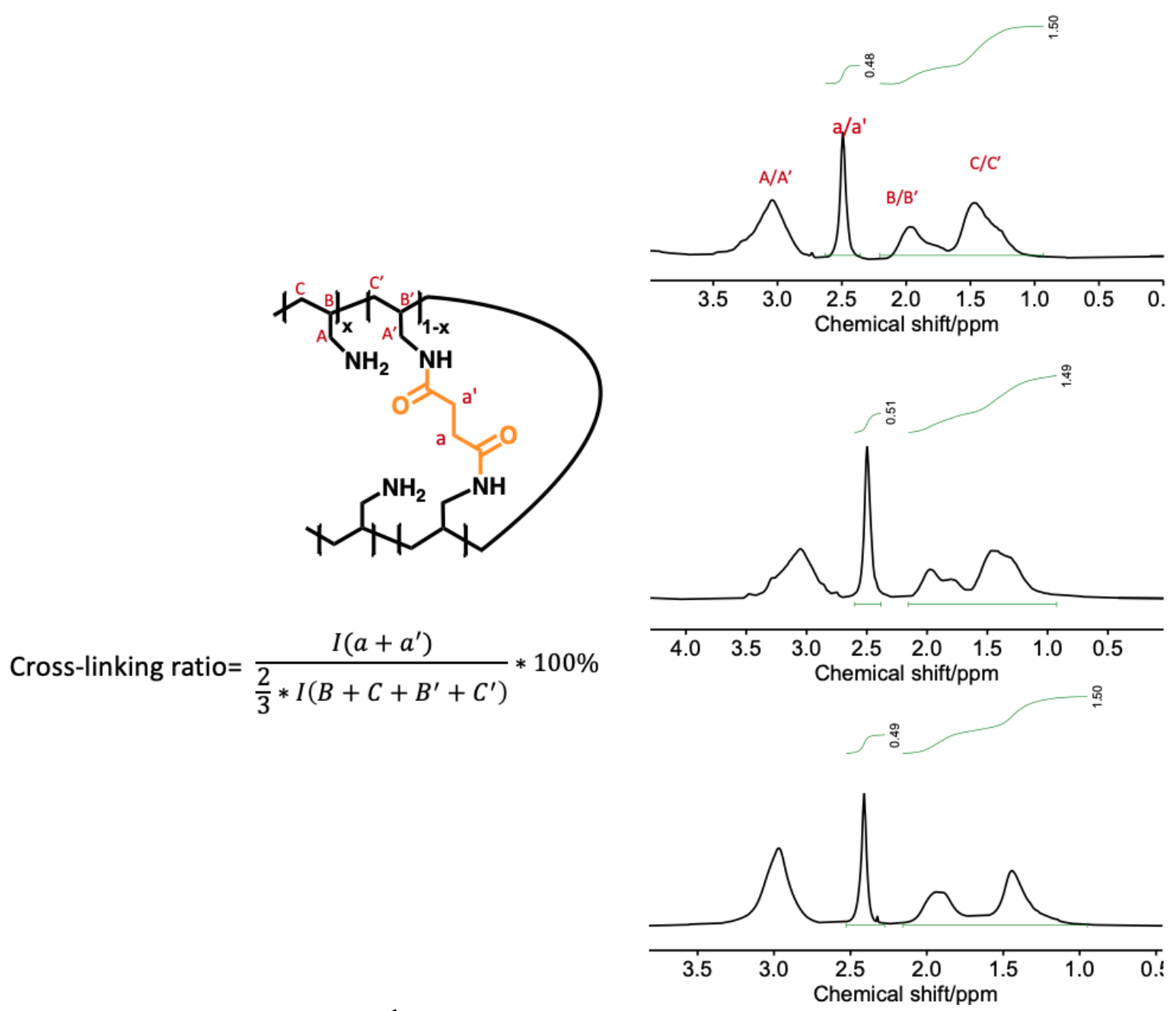

Figure S3. ${ }^{1} \mathrm{H}$ NMR of succinic acid cross-linked SCNPs 


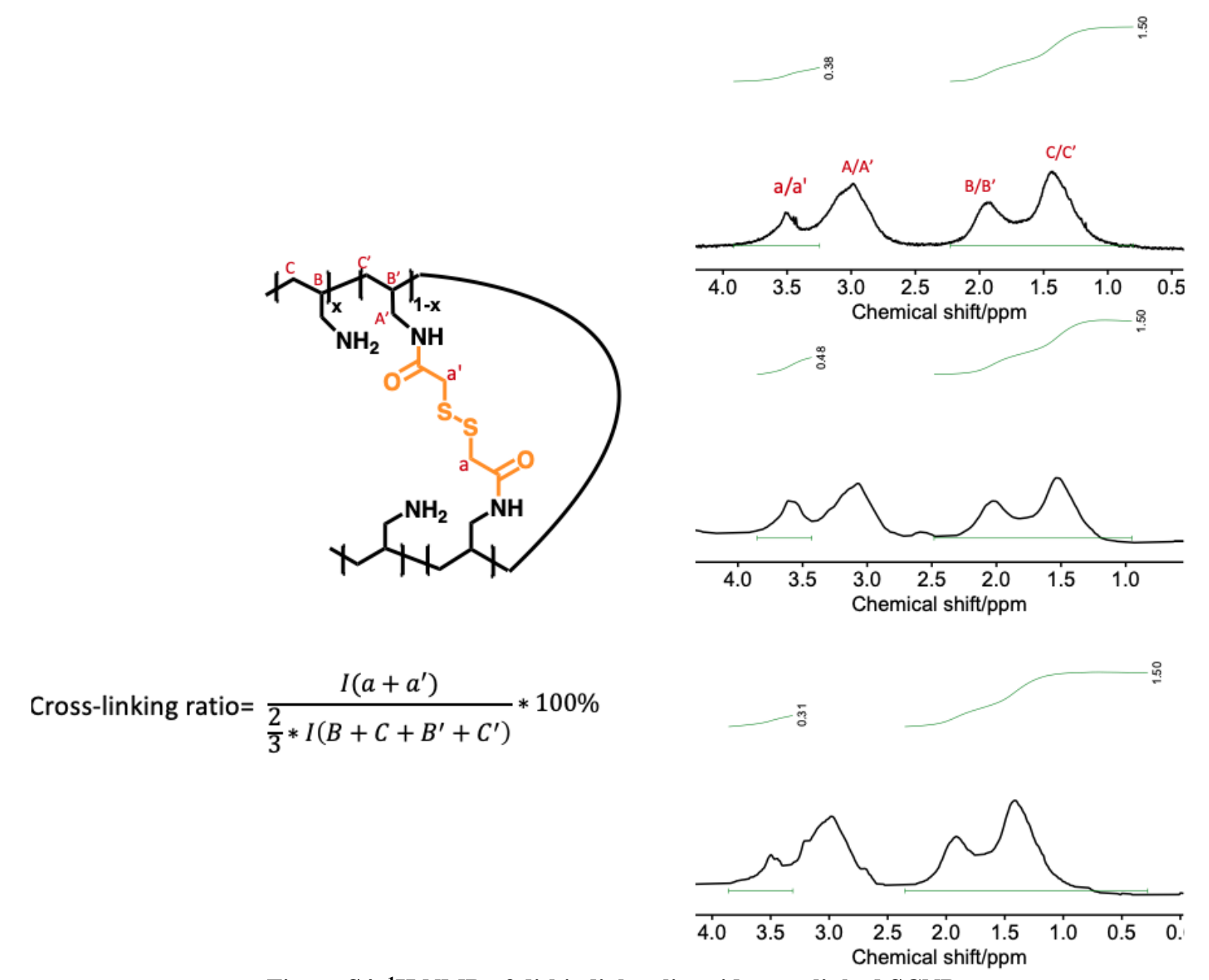

Figure S4. ${ }^{1} \mathrm{H}$ NMR of dithiodiglycolic acid cross-linked SCNPs 

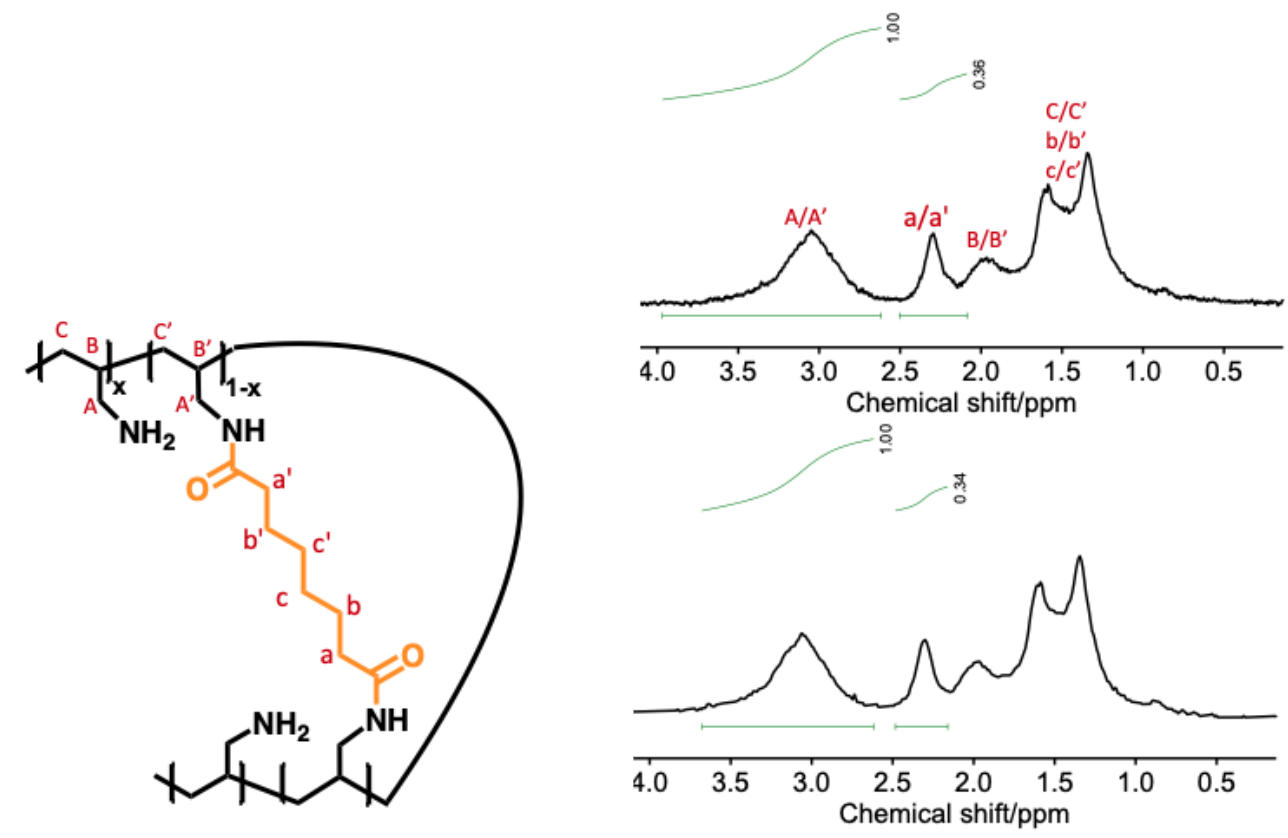

Cross-linking ratio $=\frac{I\left(a+a^{\prime}\right)}{I\left(A+A^{\prime}\right)} * 100 \%$

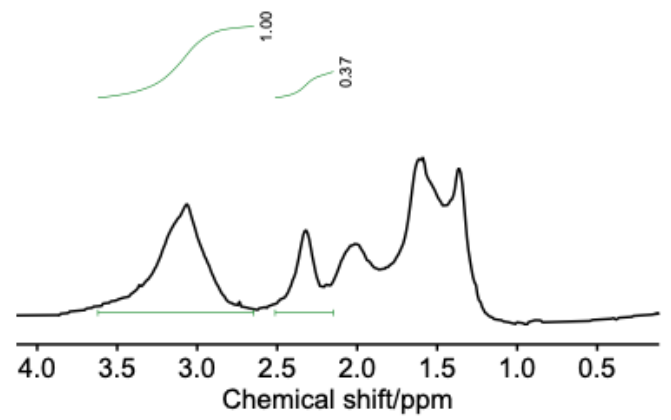

Figure S5. ${ }^{1} \mathrm{H}$ NMR of suberic acid cross-linked SCNPs 


\section{DOSY NMR}

DOSY NMR is a chromatographic tool to differentiate species by both diffusion and chemical shift. First, a magnetic field gradient is applied to spatially label the spins; then during the spin echo, the spins refocus to various degree depending on the rate of Brownian motion, thus generating a signal decay; the signal decay is fitted with the Stejskal Tanner equation to obtain the diffusion coefficient. The DOSY NMR experiments were conducted at $25^{\circ} \mathrm{C}$ on an autosampler Bruker Avance I Neo $\left({ }^{1} \mathrm{H}: 400 \mathrm{MHz}\right)$ with $5 \mathrm{~mm}$ Smart BBFO-Plusz ATMA probe. All spectra were acquired using the Bruker pulse program ledbpgp2s with a diffusion gradient of $300 \mathrm{~ms}$, a diffusion time of $5000 \mu$ s and a squared gradient ramp from 2 to $98 \%$.

Basic data processing such as baseline correction, phase correction and peak integration were performed with MestReNova (version 14.1.2). Data Fitting of the DOSY signal decay was achieved with python scripting. 


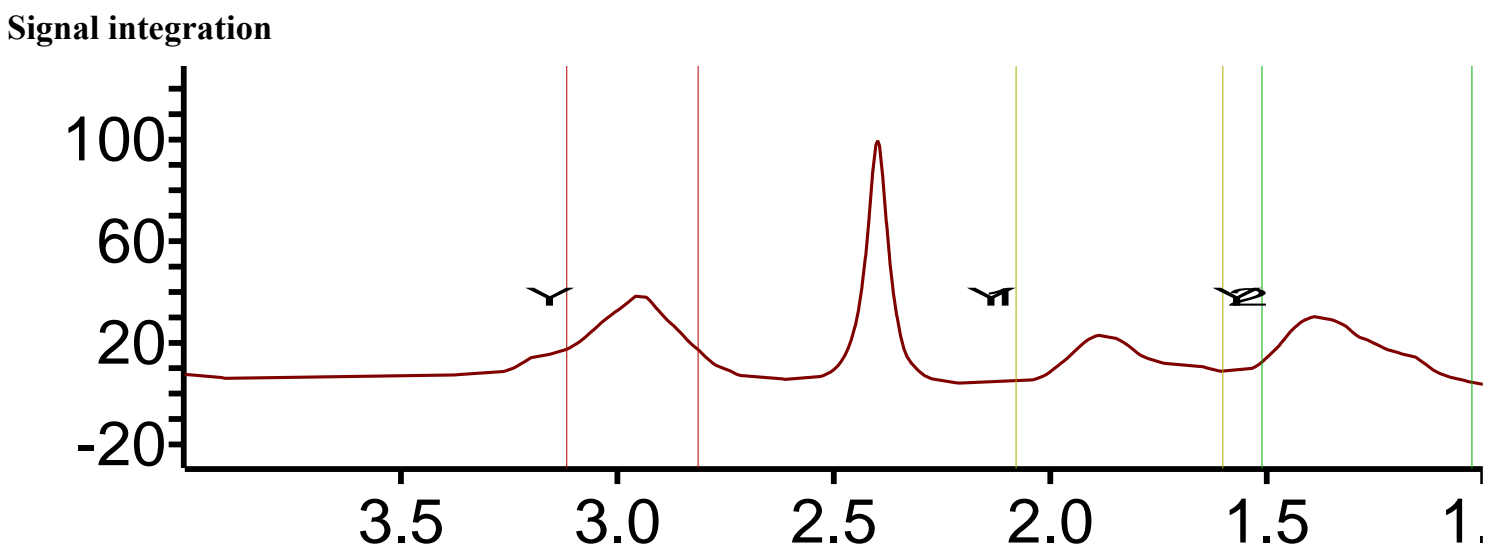

Figure S6. Signal integration example (processed in Mestrenova) 


\section{Stejskal Tanner equation}

For a better visualization of such exponential decay, the natural logarithms of normalized signal intensities were plotted against $q^{2}$ (the description of $q$ can be found under the equation) so that the slope directly indicates the rate of diffusion. Figure S7 shows the representative signal decay (proton $\mathrm{A} / \mathrm{A}^{\prime}$ as denoted in Figure 1) from each SCNP batch.

$$
\frac{I(q)}{I_{0}}=\exp \left[-D q^{2}(\Delta-\delta / 3)\right]
$$

$\mathrm{q}=\gamma \mathrm{g} \delta$

$\gamma:$ proton gyromagnetic ratio

$g$ : gradient amplitude

$\delta:$ gradient pulse duration 


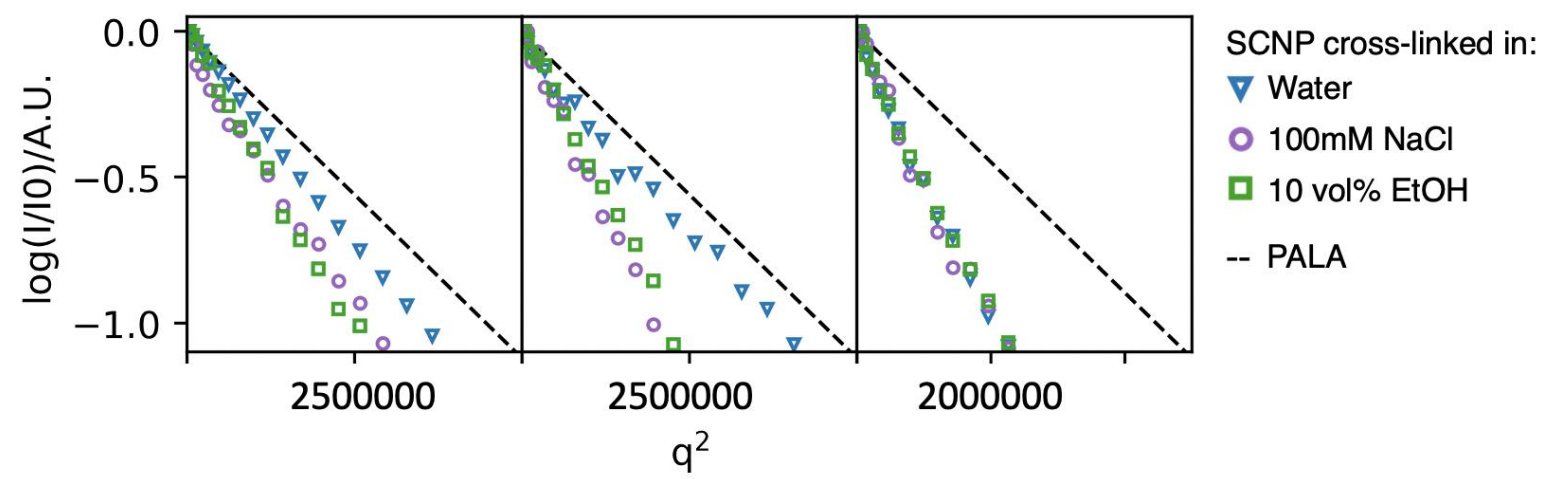

Figure S7. Scatter plots showing DOSY signal decay. (cross-linkers are succnic acids, dithiodiglycolic acids, and suberic acids, respectively in the panels from left to right) 


\section{Viscometry}

The specific viscosities (the ratio of the absolute viscosity to that of the reference fluid, herein, water) were plotted against solution concentration and the slope measures the SCNPs' contribution to the overall viscosity. The intrinsic viscosity was obtained by fitting each viscosity-concentration series with Huggins equation.

\section{Sample preparation}

To study the viscosity's dependence on the concentration of polymeric analytes, PALA/SCNPs/PAMAM G5 dendrimers were dissolved into ultrapure water. With a two-fold serial dilution, sample solutions with concentrations of $3.125,1.56,0.78,0.39,0.2 \mathrm{mg} / \mathrm{ml}$ were prepared for measurement.

\section{Measurement and Results}

Viscosity measurements were conducted on an Anton Paar Lovis 2000 rolling-ball viscometer at $20^{\circ} \mathrm{C}$. The apparent viscosity $\eta$ were corrected with the viscosity of water $\eta_{0}$ to be listed as relative viscosity as listed below.

\begin{tabular}{|c|c|c|c|c|c|c|c|c|c|c|c|}
\hline conc $(\mathrm{mg} / \mathrm{ml})$ & PALA & PAMAM G5 & $\nabla$ & ○ & 口 & $\nabla$ & 0 & 口 & $\nabla$ & 0 & 口 \\
\hline 3.125 & 1.46 & 1.02 & 1.38 & 1.22 & 1.16 & 1.36 & 1.20 & 1.14 & 1.14 & 1.15 & 1.10 \\
\hline 1.56 & 1.28 & 1.01 & 1.26 & 1.13 & 1.12 & 1.23 & 1.11 & 1.10 & 1.1 & 1.11 & 1.06 \\
\hline 0.78 & 1.15 & 1.01 & 1.16 & 1.08 & 1.05 & 1.12 & 1.06 & 1.06 & 1.06 & 1.04 & 1.03 \\
\hline 0.39 & 1.08 & 1.00 & 1.09 & 1.04 & 1.03 & 1.05 & 1.03 & 1.01 & 1.03 & 1.02 & 1.02 \\
\hline 0.2 & 1.04 & 1.00 & 1.03 & 1.02 & 1.02 & 1.00 & 1.01 & 1.02 & 1.01 & 1.01 & 1.01 \\
\hline 0 & 1.00 & 1.00 & 1.00 & 1.00 & 1.00 & 1.00 & 1.00 & 1.00 & 1.00 & 1.00 & 1.00 \\
\hline
\end{tabular}

\section{Huggins equation}

The dependence of relative viscosity on concentration can be described in the form of power series with highorder terms being negligible, due to the dilute nature of our sample solution. ([ $\eta]$ : intrinsic viscosity; $k_{h}$ : Huggins coefficient)

\section{Equation S1. Relative viscosity - concentration relationship}

$$
\eta / \eta_{0}=1+[\eta] c+k_{h}[\eta]^{2} c^{2}
$$




\section{SV-AUC}

\section{Sample preparation and experimental setting}

AUC was performed using a Beckman Optima XL-A, An-60 Ti rotor. All SCNP solutions were prepared freshly in ultrapure water to obtain final solutions that had 0.5 1.0 OD (optical density) absorbance at $230 \mathrm{~nm}$ in AUC cells (double sector titanium centerpieces with quartz windows; the optical path length is $1.2 \mathrm{~cm}$ ). All measurements were made at $20^{\circ} \mathrm{C}, 60,000$ r.p.m. (with radial step size of $0.003 \mathrm{~cm}$ ) with sufficient duration to ensure complete sedimentation. Pilot runs at varying concentrations were performed to ensure that the sedimentation and diffusion coefficients were not concentration dependent. Data ranges from 50-100 scans were chosen to represent the whole transporting process.

\section{Lamm equation}

$$
\frac{\partial c}{\partial t}=D\left(\frac{\partial^{2} c}{\partial r^{2}}+\frac{1}{r} \frac{\partial c}{\partial r}\right)-\omega^{2} s\left(r \frac{\partial c}{\partial r}+2 c\right)
$$

\section{Svedberg equation}

$$
M=\frac{R T s}{(1-\bar{v} \rho) D}
$$




\section{Data processing}

As the cross-linking densities were fixed within a comparable range for all nine SCNPs, we made the assumption that the final SCNP share the same molecular mass of $30 \pm 3 \mathrm{kDa}$. With the assumption, various values of partial specific volume were tested until the main peak obtained the assumed MW of $30 \mathrm{kDa}$. Then all the fitting parameters were transferred into the 2D model, $\mathrm{c}\left(\mathrm{s}, \mathrm{ff}_{0}\right)$, to resolve the frictional ratio.

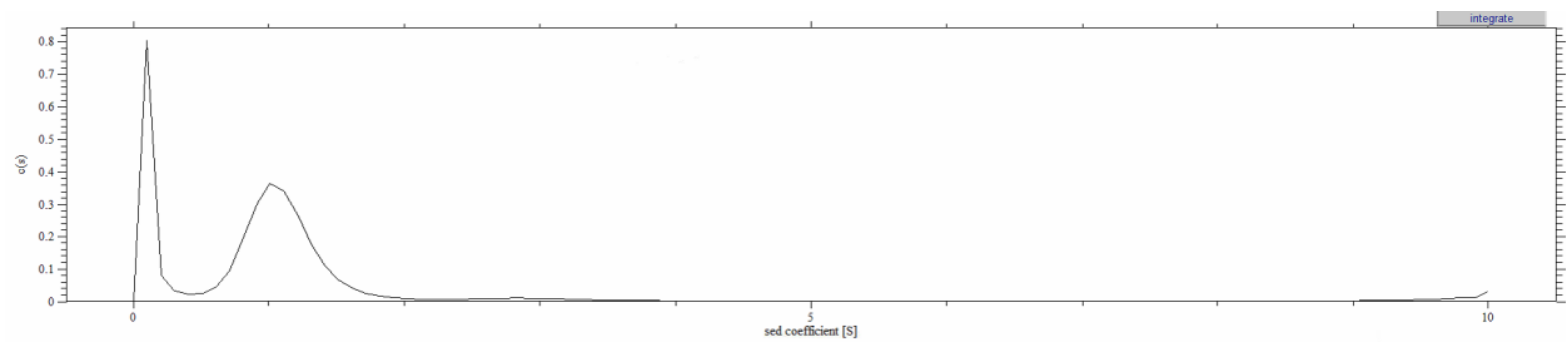

Figure S8. 1D distribution of $\mathrm{c}(\mathrm{s})$

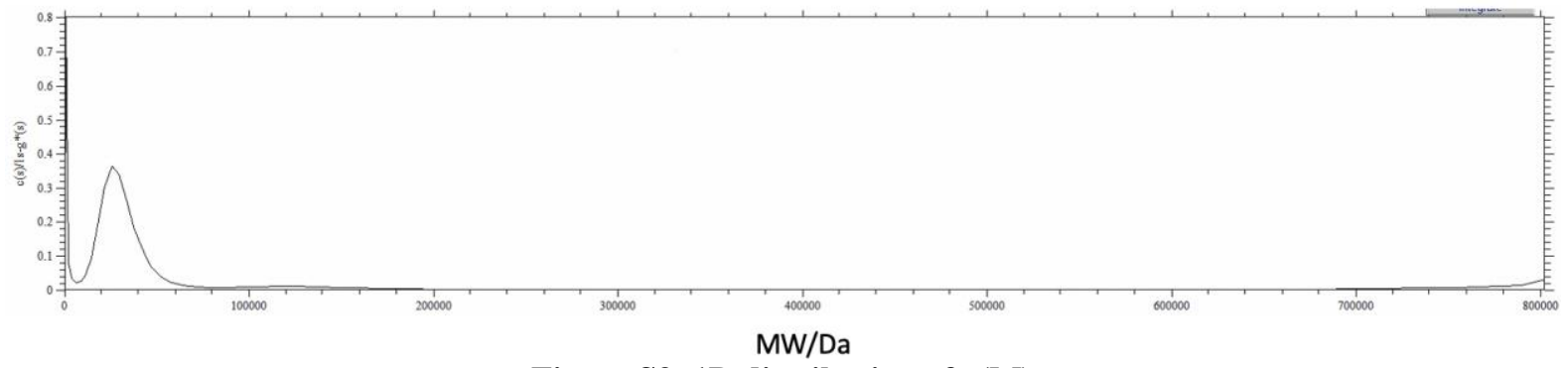

Figure S9. 1D distribution of $\mathrm{c}(\mathrm{M})$ 


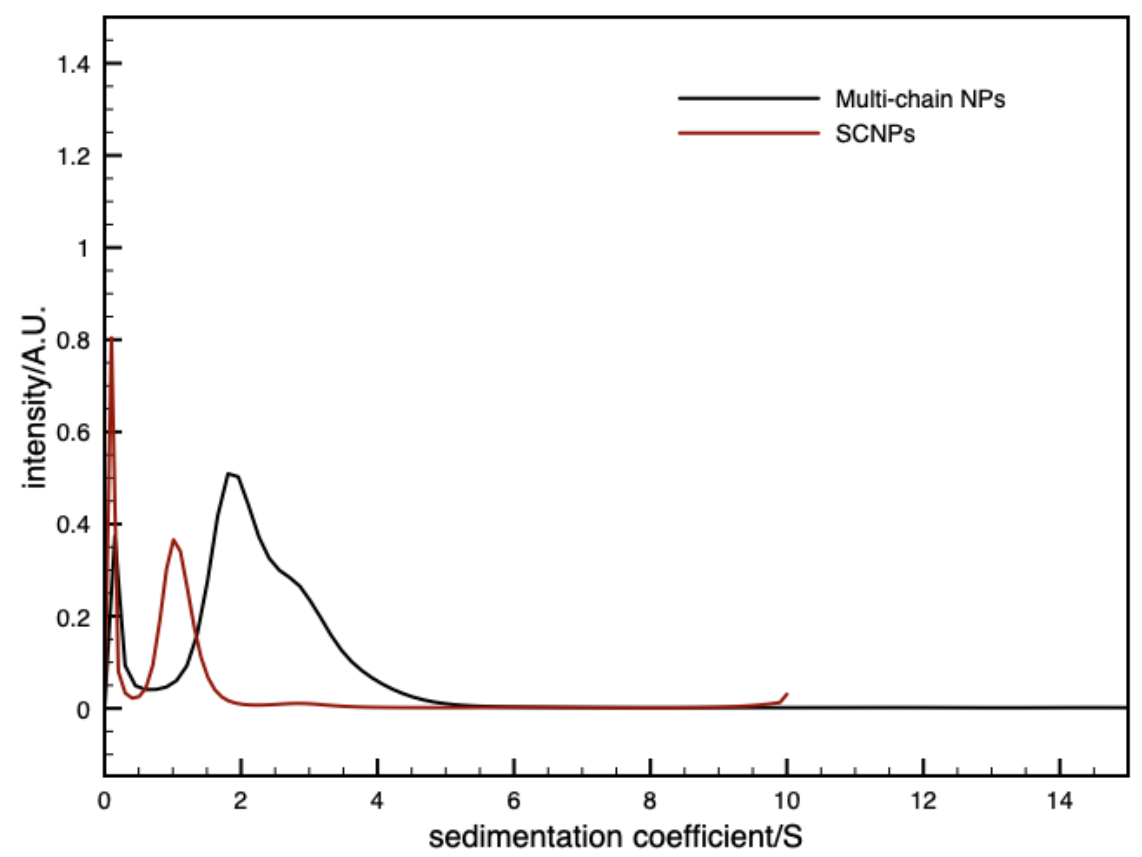

Figure S10. Distribution of sedimentation coefficient on SCNPs and multi-chain NPs (cross-linked above the critical concentration) 


\section{NMR T2 relaxation}

\section{Experimental details}

Samples for NMR spectroscopy were prepared at $2 \mathrm{~g} / \mathrm{L}$ concentrations dissolved from powders with $500 \mu \mathrm{L}$ of $100 \% \mathrm{D}_{2} \mathrm{O}$. All experiments were carried out in a Bruker $500 \mathrm{MHz}$ spectrometer equipped with a $5 \mathrm{~mm}{ }^{1} \mathrm{H},{ }^{13} \mathrm{C},{ }^{15} \mathrm{~N}$ BBI probe and an Avance Neo console. All NMR data was acquired and processed with Topspin 4 and analyzed with MestreNova 14.1.2.

${ }^{1} \mathrm{H} \mathrm{T}_{2}$ relaxation was measured using a spin echo sequence that removes $\mathrm{J}$ modulation ${ }^{2}$ using CPMG delays (in s) of $0.0020155,0.004031,1.03194,0.0060465,0.008062,0.0100775,0.0141085,0.0181395,0.024186,0.028217$, $0.032248,0.04031,0.048372,0.056434,0.064496,0.072558,0.08062,0.088682,0.096744,0.104806,0.112868$, $0.12093,0.128992,0.193488,0.257984,0.386976,0.515968,0.773952,1.03194,0.0020155,2.06387$

\section{GPC}

GPC analysis was performed using an Agilent PL-GPC 50 equipped with a refractive index detector. The mobile phase was acetate buffer at a flow of $1.0 \mathrm{~mL} / \mathrm{min}$ at $40^{\circ} \mathrm{C}$. Samples were filtered prior to analysis and results were calibrated with PEG standards. 


\section{MTS assay}

\section{Experimental protocol}

Cell cytotoxicity in this work was performed on Hela cell line with MTS assay. Hela cells were plated 12 hours before with seeding density $1.5 \times 10^{4}$ in $200 \mu \mathrm{L}$ DMEM medium supplemented with $10 \%$ fetal bovine serum (FBS) and 1\% Penicillin Streptomycin in 96-well plates. Samples were added in fixed volume $20 \mu \mathrm{L}$ with different concentrations into each well and incubated together with cells for 24 hours at $37^{\circ} \mathrm{C}$ in $5 \%$ $\mathrm{CO}_{2}$ environment. Afterwards, materials containing cell medium was removed and cells were washed with 1x phosphate-buffered saline (PBS) twice. Then $10 \mu \mathrm{L}$ MTS tetrazolium compound was added together with $90 \mu \mathrm{L}$ original DMEM medium (without FBS or Pen strep supplement) into each well and followed with incubation $\left(37^{\circ} \mathrm{C}\right.$ and $5 \% \mathrm{CO}_{2}$ ) for 4 hours. Measurement of cell proliferation was checked via absorbance at $490 \mathrm{~nm}$ with a Tecan Plate Reader. 


\section{Zeta-potential}

The charge density from cationic moieties plays a key role in determining the cytotoxicity. With comparable cross-linking densities, all SCNPs show similar zeta-potential values.

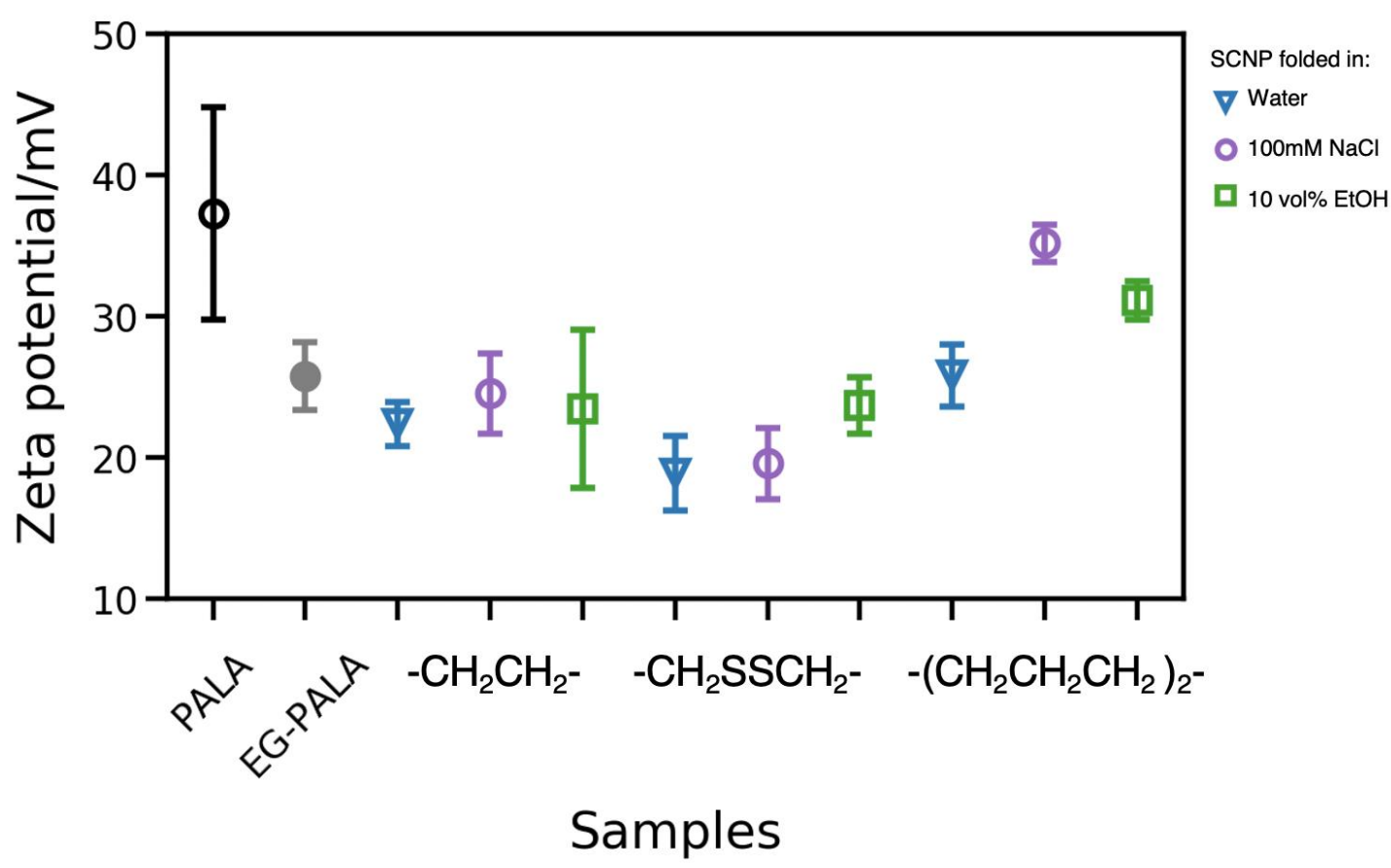

Figure S11. Scatter plot with all SCNPs and reference materials' zeta potential results (plotted as mean \pm SD) 


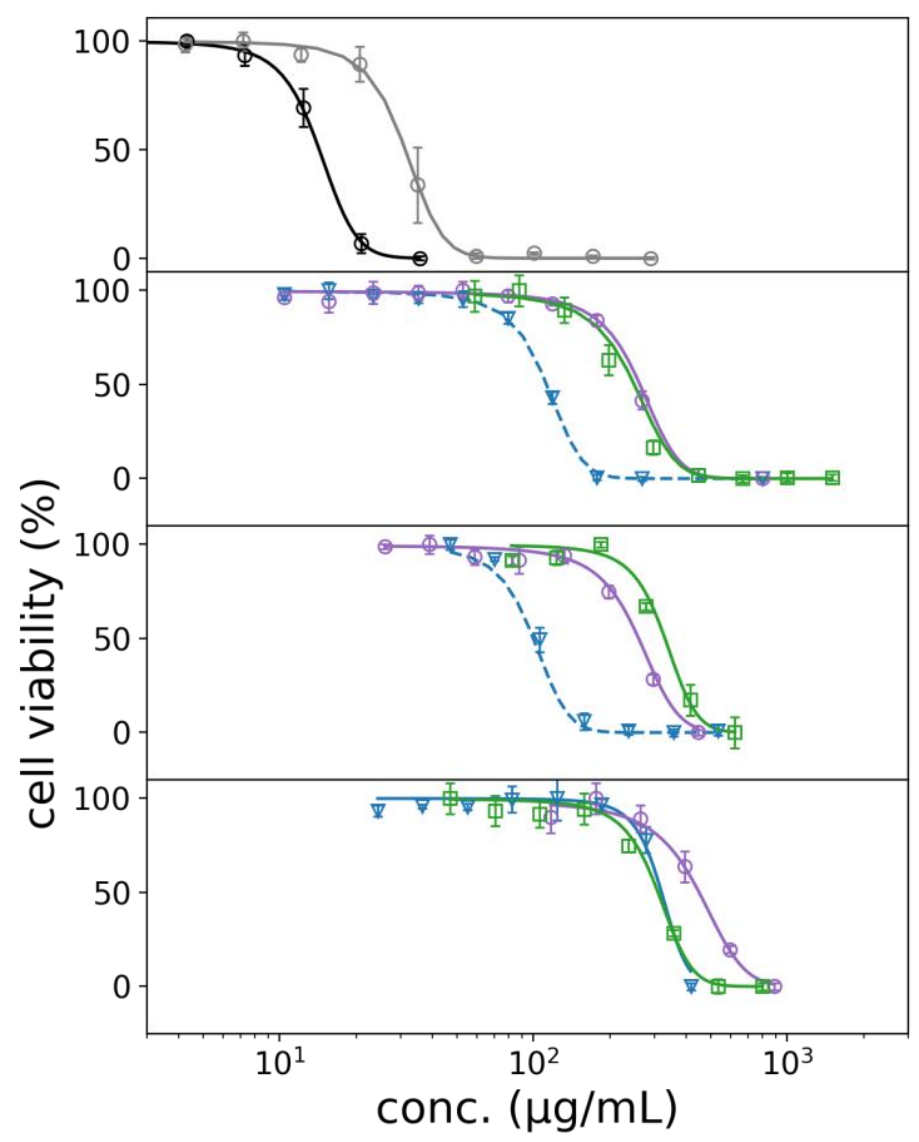

Figure S12. HeLa cell viability via MTS assay upon 24-hour incubation with all samples. (black circle: PALA; grey circle: EG-PALA). Each data point represents three independent experiments and reported as mean \pm SD. 


\section{Reference}

1. Nourse, A., Millar, D. B. \& Minton, A. P. Physicochemical characterization of generation 5 polyamidoamine dendrimers. Biopolymers 53, 316-328 (2000).

2. Aguilar, J. A., Nilsson, M., Bodenhausen, G. \& Morris, G. A. Spin echo NMR spectra without J modulation. Chem. Commun. 48, 811-813 (2011). 